\title{
Knowledge in the Performance of Newborn Hearing Screening Test of Nurses in Private Hospitals
}

\author{
Liezel Lorraine B. Olivo ${ }^{1}$ and Xerxes G. Malaga ${ }^{2}$ \\ ${ }^{1}$ Riverside Medical Center, Inc., Bacolod City, Philippines \\ ${ }^{2}$ Negros Occidental Comprehensive Health Program, Negros Occidental, Philippines
}

\begin{tabular}{l} 
Article history \\
Submitted: 23 October 2020 \\
Revised: 2 November 2020 \\
Accepted: 13 November 2020 \\
\hline Keywords \\
Nursing \\
Newborn Hearing Screening \\
Test \\
Knowledge \\
Nurses \\
Private Hospital \\
Descriptive-Comparative \\
Bacolod City
\end{tabular}

Introduction. The newborn hearing screening test is implemented before hospital discharge to initiate early identification and treatment. With a large population, lack of education, fewer professionals, and limited access to facilities, the targeted hearing screening program is implemented only to high-risk infants. The success in mandating Republic Act No. 9709, also known as the Universal Newborn Hearing Screening and Intervention Act of 2009, was enacted for the prevention, early diagnosis, and intervention of hearing loss. Healthcare professionals do not feel adequately updated with newborn hearing screening and documenting screening results. Hence, this paper describes the level of knowledge of nurses in performing newborn hearing screening tests. Likewise, it determines the difference existing between nurses' demographics and their level of knowledge. Also, it identifies nurses' level of knowledge based on areas of procedures, standards, and protocols of newborn hearing screening tests and challenges and best practices encountered.

Methods. The study utilized a descriptive and comparative research design. The descriptivecomparative research design was used to assess and compare the level of knowledge in the performance of the newborn hearing screening tests of nurses in the areas of procedures, standards, and protocols and identify the challenges and best practices encountered. The respondents of the study were the 97 pediatric nurses of private hospitals in Negros Occidental who provided direct care to newborns. Total enumeration was used to identify the actual respondents. A researchermade test questionnaire adapted from the Manual of Operation of the Republic Act No. 9709 (MOP) was utilized. Mean and Standard Deviation were used to assess the level of knowledge of nurses. Frequency count and percentage distribution were used to identify the challenges and best practices. Mann Whitney $U$ test was used to determine the significant difference in the level of knowledge according to variables age, years of service, and hospital affiliation.

Results. The findings of the study revealed that the level of knowledge of nurses in private hospitals is generally high in performing newborn hearing screening tests in the areas of procedures, standards, and protocols. There are challenges and best practices encountered by nurses in conducting the procedure. However, when classified under the guidelines, results showed that nurses are still functional in being efficient in their patients' sustainable screening. As to the difference between demographics and level of knowledge when grouped according to age and hospital affiliation, the findings reveal no significant difference. On the other hand, there is a significant difference in nurses' level of knowledge in performing newborn hearing screening tests when grouped according to years of service.

Conclusion. The level of knowledge in the performance of newborn hearing screening tests of nurses in private hospitals in Bacolod City is high, which indicates that the best quality care is given to patients. It shows that nurses' performance passed the high-quality standard of care. The nurses are focused on hospital procedures in providing care to newborns. Moreover, nurses in tertiary hospitals are highly motivated to perform such procedures, whether in a resource-limited setting and despite enormous challenges. 
Practical Value of the Paper. The study significantly contributes to the few existing literature on nurses' level of knowledge in performing newborn hearing screening tests. In addition, the findings of the study provided baseline data to hospital administrators and nurses in developing a training program and standard operating procedure that will be integrated into the hospital policy manual to enhance or improve nurses' performance in patient safety practices.

\section{References}

Abdullah, N. A., Zakaria, M. N., Majid, A. H., Hamzah, S., Ismail, A. I., \& Mukari, S. Z. M. S. (2017). Determinants of Employees' Awareness for Universal Newborn Hearing Screening: Evidence from Malaysia. Journal of Economic \& Management Perspectives, 11(4), 702-711.

Alshawi, Y. A., Al-Gazlan, N., Alrawaf, F., \& Almuhawas, F. (2019). Value of Newborn Hearing Screening on Early Intervention in the Saudi Population and Review of International Records. Cureus, 11(10).

Bellcross, C. A., Harmond, L., Floyd-Browning, P., \& Singh, R. (2015, December). Infrastructure and Educational Needs of Newborn Screening Short-Term Follow-Up Programs within the Southeast Regional Newborn Screening \& Genetics Collaborative: A Pilot Survey. In Healthcare (Vol. 3, No. 4, pp. 964-972). Multidisciplinary Digital Publishing Institute.

Galhotra, A., \& Sahu, P. (2019). Challenges and solutions in implementing a hearing screening program in India. Indian Journal of Community Medicine: Official Publication of Indian Association of Preventive \& Social Medicine, 44(4), 299.

Padilla, C. D., \& Cutiongco-de la Paz, E. M. (2016). Genetics and genomic medicine in the Philippines. Molecular genetics \& genomic medicine, 4(5), 494.

Pitathawatchai, P., Khaimook, W., \& Kirtsreesakul, V. (2019). Pilot implementation of newborn hearing screening program at four hospitals in southern Thailand. Bulletin of the World Health Organization, 97(10), 663.

Que, M. H. B., \& Reyes-Quintos, M. R. T. (2018). Evaluation of the Newborn Hearing Screening Program in The Medical City Based on Joint Commission on Infant Hearing (JCIH) 2007 Position Statement Quality Indicators. Philippine Journal of Otolaryngology-Head and Neck Surgery, 33(1), 21-24.

Roberts, C., \& Jones, A. L. (2017). Measuring nurses' knowledge and understanding of universal newborn hearing screenings. Journal of Early Hearing Detection and Intervention, 2(2), 38-47.

Singh, M. (2014). The art, science, and philosophy of newborn care. The Indian Journal of Pediatrics, 81(6), 552-559.

Vishwakarma, C., Mathur, R., Vishwakarma, R., Jindal, S., Sharma, P., \& Sinha, V. (2015). Universal hearing screening vs. targetted hearing screening: Make a choice. Indian Journal of Otology, 21(3), 179.

\section{Correspondence:}

Liezel Lorraine B. Olivo [olivo.l@yahoo.com]

http://orcid.org/0000-0002-5591-9685 